\title{
A remark on the theory of semi-functors
}

\author{
R. HOOFMAN ${ }^{\dagger}$ and I. MOERDIJK \\ ${ }^{\dagger}$ University of Amsterdam \\ Email: raymondQfwi.uva.nl \\ ${ }^{\ddagger}$ University of Utrecht \\ Email: moerdijk@math.ruu.n1
}

Received 17 May 1993; revised 28 March 1994

By establishing an appropriate equivalence, we observe that the theory of semi-functors can be fully embedded in the theory of (ordinary) functors. As a result, standard properties and constructions on functors extend automatically to semi-functors.

\section{Introduction}

Categories and functors play a central role in the semantics of various formal languages. For example, it is well known that extensional typed lambda calculi are essentially equivalent to cartesian closed categories (see e.g. Lambek and Scott (1974)).

In order to develop a similar categorical semantics for non-extensional typed lambda calculi, S. Hayashi introduced in Hayashi (1985) the notion of a semi-functor between categories. He also developed in loc. sit. the theory of semi-adjunctions and semi cartesian closed categories, and showed that the latter correspond to non-extensional typed lambda calculi. He also showed how the theory of semi-functors provides a natural approach to the 'extensionalization' of models of the lambda calculus. Later on, semi-functors were studied in the context of linear logic and the second-order lambda calculus: see e.g. Hoofman (1992), Hoofman and Schellinx (1991), Jacobs (1991), Martini (1987, 1992), Román (1989). A systematic exposition of the theory of semi-functors is given in Hoofman (1993a).

Although the introduction of semi-functors at first sight appears to be a proper extension of ordinary category theory, we observe in this paper that the theory of semi-functors can be fully embedded into the theory of (ordinary) functors. More specifically, we establish an appropriate equivalence between, on the one hand, the theory of categories, semi-functors and natural transformations between such semi-functors, and, on the other hand, the theory of Karoubi-complete categories, ordinary functors and ordinary natural transformations. A precise statement of this equivalence occurs as our main Theorem 1 below.

As a consequence of this result, many standard properties and constructions of functors extend automatically to semi-functors (see Section 3). In particular, many results of Hoofman (1993a) are immediate consequences of our main theorem.

In Section 4 we investigate the relation of our approach to the work of Koymans (Koymans 1982), and in the final section we will present an extension of our main theorem 
to the context of fibred (or indexed) categories. We are motivated by the fact that such fibred categories (with appropriate properties) provide models of second-order languages (see e.g. Jacobs (1991), Seely (1987)). Indeed, in Hoofman and Moerdijk (1994) we will show that this extension to fibred categories provides a method for the extensionalization of second-order calculi.

\section{Semi-functors and the Karoubi envelope}

In this preliminary section we review some basic definitions. We assume that the reader is familiar with the notions of 2-category, 2-functor, etc. (a basic reference is Kelly and Street (1974)). For a 2-category C, the category obtained by forgetting the 2-cells of $\mathrm{C}$ will again be denoted by $C$.

Let $C$ and D be categories. Recall from Hayashi (1985), Hoofman (1993a) and the introduction, that a semi-functor $F: C \rightarrow D$ maps objects, respectively arrows, in $C$ to objects, respectively arrows, in D, preserving domain, codomain and composition. Hence a semi-functor is defined in the same way as a functor, except that a semi-functor need not preserve identities. For semi-functors $F$ and $G: C \rightarrow D$, a natural transformation between semi-functors $\alpha: F \rightarrow G$ is a family $\left(\alpha_{C}: F C \rightarrow G C \mid C \in C\right.$ ) of arrows in the category D, satisfying the two conditions

$1 \alpha_{D} \circ F(f)=G(f) \circ \alpha_{C}$,

$2 \alpha_{C} \circ F\left(i d_{C}\right)=\alpha_{C}$.

Semi-functors and natural transformations between semi-functors can be composed in the obvious ways, thus yielding a 2-category denoted by Cat $_{s}$. The usual 2-category Cat of categories, functors and natural transformations is a subcategory of Cat , full on 2-cells.

Recall that an idempotent in a category $C$ is an arrow $p: C \rightarrow C$ in $C$ such that $p \circ p=p$. A splitting of $p$ is a triple $\langle R, r, s\rangle$, where $r: C \rightarrow R$ and $s: R \rightarrow C$ are arrows satisfying $r \circ s=i d_{R}$ and $s \circ r=p$. Such a splitting of a given idempotent is unique up to isomorphism. One says that a category $\mathrm{C}$ has splittings (or that idempotents split in $\mathrm{C}$, or that $\mathrm{C}$ is Karoubi complete) iff a splitting exists for every idempotent arrow. (Observe that we do not require a 'canonical' choice of splittings; see Section 4 below.) We write Cat ${ }_{i}$ for the full 2-subcategory of Cat with the categories that have splittings as objects. Thus

$$
\mathrm{Cat}_{i} \hookrightarrow \text { Cat } \hookrightarrow \mathrm{Cat}_{s}
$$

The Karoubi envelope $\mathscr{K} \mathrm{C}$ of a category $\mathrm{C}$ is obtained by freely adding splittings for idempotents in $\mathrm{C}$ : objects of $\mathscr{K} \mathrm{C}$ are pairs $\langle C, p\rangle$, where $p: C \rightarrow C$ is an idempotent in $\mathrm{C}$, while arrows $f:\langle C, p\rangle \rightarrow\langle D, q\rangle$ in $\mathscr{K} \mathrm{C}$ are arrows $f: C \rightarrow D$ in $\mathrm{C}$ with the property that $q \circ f \circ p=f$. (Note that the identity arrow on $\langle C, p\rangle$ is not the identity arrow id $d_{C}$ of $\mathrm{C}$ but $p:\langle C, p\rangle \rightarrow\langle C, p\rangle$.) It is easy to see that any idempotent $f:\langle C, p\rangle \rightarrow\langle C, p\rangle$ in $\mathscr{K} \mathrm{C}$ splits, via the object $R=\langle C, f\rangle$.

If $F: \mathrm{C} \rightarrow \mathrm{D}$ is a semi-functor, one obtains an ordinary functor $\mathscr{K}(F): \mathscr{K C} \rightarrow$ $\mathscr{K} \mathrm{D}$ defined on objects by $\mathscr{K}(F)\langle C, p\rangle=\langle F C, F(p)\rangle$, while on arrows $\mathscr{K}(F)(f)=F(f)$. Similarly, a semi natural transformation $\alpha: F \rightarrow G$ between semi-functors yields an 
ordinary natural transformation $\mathscr{K}(\alpha): \mathscr{K}(F) \rightarrow \mathscr{K}(G)$, with components $\alpha_{\langle C, p\rangle}=\alpha_{C} \circ$ $F(p)\left(=G(p) \circ \alpha_{C}\right)$. In this way one obtains a 2-functor

$$
\mathscr{K}: \mathrm{Cat}_{s} \rightarrow \mathrm{Cat}_{i} .
$$

In Freyd and Scedrov (1989) it is observed that when viewed as a functor $\mathscr{K}:$ Cat $_{s} \rightarrow$ Cat, the Karoubi envelope is right adjoint to the inclusion Cat $\hookrightarrow \mathrm{Cat}_{s}$ : see also Hoofman (1993a).

\section{The main observation}

Our main result is the following:

Theorem 1. The Karoubi envelope $\mathscr{K}$ defines an equivalence of 2-categories

$$
\mathscr{K}: \text { Cat }_{s} \stackrel{\simeq}{\rightarrow} \text { Cat }_{i} \text {. }
$$

As we shall see, this theorem is not difficult to prove. Nonetheless, it is fundamental for the theory of semi-functors, since it allows us to extend properties of functors and natural transformations in a systematic way to semi-functors and natural transformations between semi-functors. Some instances are mentioned below.

For the proof of the theorem, first recall that a functor $F: C \rightarrow D$ is an (ordinary) equivalence of categories iff $F$ induces an isomorphism from $\mathrm{C}\left(C, C^{\prime}\right)$ to $\mathrm{D}\left(F C, F C^{\prime}\right)$, and, moreover, $F$ is surjective up to isomorphism (in the sense that for each $D \in D$ there exists $C \in \mathrm{C}$ such that $F C \cong D$ ). Uniformly substituting 'equivalent' for 'isomorphic' in this definition yields the notion of an equivalence of 2-categories. In detail:

Definition 2. Let $C, D$ be 2-categories. A 2-functor $F: C \rightarrow D$ is an equivalence of 2-categories (and $\mathrm{C}$ and $\mathrm{D}$ are 2-equivalent) iff the following conditions hold:

1 For any objects $C, C^{\prime} \in \mathrm{C}$ the 2-functor $F$ restricted to $\mathrm{C}\left(C, C^{\prime}\right) \rightarrow \mathrm{D}\left(F C, F C^{\prime}\right)$ is an ordinary equivalence of categories.

2 The 2-functor $F$ is surjective up to equivalence: for each $D \in D$ there exists $C \in C$ such that $F C \simeq D$ (i.e., there are one-cells $f: F C \rightarrow D, g: D \rightarrow F C$, and invertible 2-cells $f \circ g \cong 1_{D}$ and $g \circ f \cong 1_{F C}$ ).

Now we give the proof of Theorem 1:

Proof. For any category $\mathrm{C}$, there is an evident functor $\eta_{\mathrm{C}}: \mathrm{C} \rightarrow \mathscr{K}(\mathrm{C})$ given on objects by $\eta_{C}(C)=\left\langle C, i d_{C}\right\rangle$. This functor is full and faithful. Furthermore, it is well known that $\eta_{C}$ is an equivalence of categories when idempotents split in $C$. (Indeed, if $\langle R, r, s\rangle$ is a splitting for $\langle C, p\rangle$, then $r$ and $s$ define an isomorphism $\eta_{C}(R) \cong\langle C, p\rangle$ in $\mathscr{K}$ C.) This shows that the 2-functor $\mathscr{K}: \mathrm{Cat}_{s} \rightarrow \mathrm{Cat}_{i}$ is surjective up to equivalence.

The 'forgetful' semi-functor $\varepsilon_{\mathrm{C}}: \mathscr{K}(\mathrm{C}) \rightarrow \mathrm{C}$ runs in the opposite direction of $\eta_{\mathrm{C}}$, and is defined on objects by $\varepsilon_{C}\langle C, p\rangle=C$. To complete the proof of the theorem, we now show that for any categories $C$ and $D$, the Karoubi envelope gives an (ordinary) equivalence of categories $\mathscr{K}: \mathrm{Cat}_{s}(\mathrm{C}, \mathrm{D}) \stackrel{\sim}{\rightarrow} \mathrm{Cat}_{i}(\mathscr{K} \mathrm{C}, \mathscr{K} \mathrm{D})$.

First, this restriction of $\mathscr{K}$ to hom-sets is surjective up to isomorphism: for any functor $F: \mathscr{K C} \rightarrow \mathscr{K} \mathrm{D}$ it can easily be checked that $F \cong \mathscr{K} \bar{F}$, where $\bar{F}$ is the semi-functor $\varepsilon_{\mathrm{D}} \circ F \circ \eta_{\mathrm{C}}: \mathrm{C} \rightarrow \mathrm{D}$. 
Next, we observe that the Karoubi envelope $\mathscr{K}$ restricted to hom-sets is full and faithful. Indeed, if $F, G: \mathrm{C} \rightarrow \mathrm{D}$ are semi-functors, the operation $\operatorname{Nat}(F, G) \rightarrow \operatorname{Nat}(\mathscr{K}(F), \mathscr{K}(G))$ : $\alpha \mapsto \mathscr{K}(\alpha)$ from natural transformations $F \rightarrow G$ to natural transformations $\mathscr{K}(F) \rightarrow \mathscr{K}(G)$ is a bijection. Its inverse is $\operatorname{Nat}(\mathscr{K}(F), \mathscr{K}(G)) \rightarrow \operatorname{Nat}(F, G): \beta \mapsto \beta \cdot \eta_{C}$, where the components of $\beta \cdot \eta_{\mathrm{C}}$ are given by $\left(\beta \cdot \eta_{\mathrm{C}}\right)_{C}=\beta_{\langle C, i d\rangle}$, for any object $C \in \mathrm{C}$.

Note that the inclusion $\mathrm{Cat}_{i} \hookrightarrow \mathrm{Cat}_{s}$ is a quasi inverse for $\mathscr{K}: \mathrm{Cat}_{s} \rightarrow \mathrm{Cat}_{i}$. In other words, any category $\mathrm{C}$ is semi-equivalent to its Karoubi envelope $\mathscr{K} \mathrm{C}$ via $\eta_{\mathrm{C}}: \mathrm{C} \rightarrow \mathscr{K} \mathrm{C}$, $\varepsilon_{\mathrm{C}}: \mathscr{K} \mathrm{C} \rightarrow \mathrm{C}$.

A large part of the theory of semi-functors presented in Hoofman (1993a) can be viewed as an immediate application of Theorem 1. For example, when one defines a semi-adjunction between semi-functors $F: C \rightarrow D, G: D \rightarrow C$ in the standard way in the language of the 2-category $\mathrm{Cat}_{s}$ (see Kelly and Street (1974)), $F$ is left semi-adjoint to $G$ iff $\mathscr{K}(F): \mathscr{K} \mathrm{C} \rightarrow \mathscr{K} \mathrm{D}$ is left adjoint to $\mathscr{K}(G)$. Furthermore, all well-known categorical properties of adjoint functors immediately transport along the equivalence $\mathrm{Cat}_{i} \simeq \mathrm{Cat}_{s}$ to give the same properties of semi-adjoint semi-functors. (For example, semi-adjoints are unique up to semi natural isomorphism, semi-adjunctions compose, etc.)

The same principal applies to other properties that can be expressed in the language of 2-categories. Thus a category $\mathrm{C}$ has semi-products iff $\mathscr{K} \mathrm{C}$ has products. (Indeed, the existence of a right (semi-)adjoint to the diagonal $\mathrm{C} \rightarrow \mathrm{C} \times \mathrm{C}$ is a 2-categorical property of

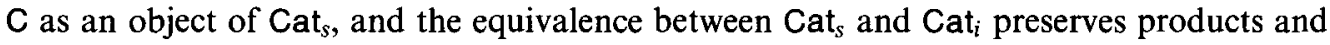
diagonals.) Similarly, a category is semi cartesian closed iff $\mathscr{K} \mathrm{C}$ is cartesian closed. As a last example, one can develop a theory of semi-monads and semi-algebras by transporting the usual theory of algebras and monads (formulated for objects in a 2-category in Kelly and Street (1974) and Street (1972)) along the equivalence of Theorem 1. The notion of a semi-algebra finds an application in Hoofman (1993b).

\section{The Karoubi envelope as a monad}

The main purpose of this section is to point out some relations with the work of Koymans (Koymans 1982). We assume that the reader is familiar with the notions of a monad, the Kleisli category construction, and the Eilenberg-Moore category (a basic reference is Mac Lane (1971)).

First, we observe that the Karoubi envelope yields a monad on the category Cat. Indeed, the functor $\mathscr{K}:$ Cat $\rightarrow$ Cat (i.e., Cat $\rightarrow$ Cat $_{i} \hookrightarrow$ Cat) comes equipped with natural transformations $\eta: I d \rightarrow \mathscr{K}$ and $\mu: \mathscr{K}^{2} \rightarrow \mathscr{K}$ as follows: define $\eta$ as in the proof of Theorem 1, and take $\mu_{\mathrm{C}}\langle\langle C, p\rangle, f\rangle=\langle C, f\rangle$. (Actually, $\mathscr{K}$ is a 2-comonad on the 2-category Cat.)

Next we consider the Kleisli category of the Karoubi monad. Recall that the objects in the Kleisli category are the same as in Cat, viz. categories, whereas arrows $F: C \rightarrow D$ in the Kleisli category are functors $F: C \rightarrow \mathscr{K} \mathrm{D}$ in Cat. We observe that each such functor $F$ gives rise to a semi-functor $\eta_{\mathrm{D}} \circ F: \mathrm{C} \rightarrow \mathrm{D}$, while each semi-functor $G: \mathrm{C} \rightarrow \mathrm{D}$ yields a functor $\mathscr{K}(G) \circ \varepsilon_{\mathscr{K}} \mathrm{D}: \mathrm{C} \rightarrow \mathrm{D}$. As this defines a one-to-one correspondence between arrows in $\mathrm{Cat}_{s}$ and arrows in the Kleisli category of $\mathscr{K}$, it follows that the last category is actually isomorphic to $\mathrm{Cat}_{s}$. 
Proposition 3. The category $\mathrm{Cat}_{s}$ of categories and semi-functors is isomorphic to the Kleisli category of the Karoubi $\operatorname{monad}\langle\mathscr{K}, \eta, \mu\rangle$ on Cat.

We remark that this isomorphism can also be extended to an isomorphism of 2-categories. As an easy consequence of the proposition, standard monad theory tells us that there exists a (2-)adjunction between Cat and $\mathrm{Cat}_{s}$, which is of course the adjunction mentioned in Section 2.

Now consider the Eilenberg-Moore category (the category of algebras) of the Karoubi monad. Recall that the objects of this category are functors of the form $F: \mathscr{K C} \rightarrow \mathrm{C}$ that satisfy $F \circ \eta_{\mathrm{C}}=I d_{\mathrm{C}}$ and $F \circ \mu_{\mathrm{C}}=F \circ \mathscr{K}(F)$. An arrow $H:(F: \mathscr{K} \mathrm{C} \rightarrow \mathrm{C}) \rightarrow(G: \mathscr{K} \mathrm{D} \rightarrow \mathrm{D})$ consists of a functor $H: \mathrm{C} \rightarrow \mathrm{D}$ that satisfies the requirement $G \circ \mathscr{K}(H)=H \circ F$. The Eilenberg-Moore category can be described as the category Cat ${ }_{i c}$ of categories with chosen splittings and splitting-preserving functors, defined as follows:

Definition 4. A category $\mathrm{C}$ has chosen splittings if there is given a family $\left(R_{p}, r_{p}, s_{p} \mid\right.$ $p$ idempotent in $\mathrm{C}$ ), where each triple $\left\langle R_{p}, r_{p}, s_{p}\right\rangle$ is a splitting of $p$, satisfying the following requirements:

$1 R_{i d_{A}}=A$,

$2 r_{i d_{A}}=i d_{A}$,

$3 s_{i d_{A}}=i d_{A}$.

Moreover, for each pair of idempotents $p, q$ satisfying $p \circ q \circ p=p$,

$4 R_{r_{p} \circ q \circ s_{p}}=R_{q}$,

$5 r_{r_{p} \circ q \circ s_{p}} \circ r_{p} \circ q=r_{q}$,

$6 q \circ s_{f} \circ s_{r_{p} \circ q \circ s_{p}}=s_{q}$.

If, also, D has chosen splittings denoted by $\left\langle R_{p}^{\prime}, r_{p}^{\prime}, s_{p}^{\prime}\right\rangle$, the functor $H: \mathrm{C} \rightarrow \mathrm{D}$ is said to preserve chosen splittings iff $H\left(R_{p}\right)=R_{H(p)}^{\prime}, H\left(r_{p}\right)=r_{H(p)}^{\prime}$, and $H\left(s_{p}\right)=s_{H(p)}^{\prime}$. For splitting preserving functors $H, K: \mathrm{C} \rightarrow \mathrm{D}$, a natural transformation $\alpha: H \rightarrow K$ is said to preserve chosen splittings iff

$$
r_{K(p)}^{\prime} \circ \alpha_{A} \circ H(p) \circ s_{H(p)}^{\prime}=\alpha_{R_{p}} \text {. }
$$

The notion from Koymans (1982) of a category with explicit splittings differs slightly from the notion introduced here. Specifically, in the definition of a category with explicit splittings, requirement (5) above is replaced by the somewhat stronger requirement

$$
r_{r_{p} \circ q \circ S_{p}} \circ r_{p}=r_{q}
$$

with the analogous replacement for requirement (6).

Let $\mathrm{Cat}_{i c}$ denote the (2-)category of categories with chosen splittings and splitting preserving functors. Similarly, Cat ${ }_{i e}$ denotes the full (2-)subcategory of Cat ${ }_{i c}$ of categories with explicit splittings.

Proposition 5. The Eilenberg-Moore category of the Karoubi monad on Cat is isomorphic to the category Cat ${ }_{i c}$ of categories with chosen splittings.

Proof. Each chosen splitting on $\mathrm{C}$ determines an algebra $F: \mathscr{K} \mathrm{C} \rightarrow \mathrm{C}$ as follows. Let $\left(R_{p}, r_{p}, s_{p} \mid p\right.$ idempotent in $\left.\mathrm{C}\right)$ be a family of chosen splittings on $\mathrm{C}$, then take $F\langle C, p\rangle=R_{p}$ and $F(f:\langle C, p\rangle \rightarrow\langle D, q\rangle)=r_{q} \circ f \circ s_{p}$. The other way round, algebras $F: \mathscr{K C} \rightarrow \mathrm{C}$ give rise to chosen splittings: take $R_{p}=F\langle C, p\rangle, r_{p}=F(p)$, and $s_{p}=F(p)$. It is easy to 
check that this yields a one-to-one correspondence between algebras and chosen splittings. Furthermore, splitting preserving functors correspond to morphisms between algebras.

As in the case of Proposition 3, the isomorphism of Proposition 5 can be extended to an isomorphism of 2-categories. Furthermore, by standard monad theory we find an adjunction between Cat and $\mathrm{Cat}_{i c}$, which restricts to the (2-)adjunction described in Koymans (1982) between Cat and Cat ie $_{\text {. }}$

\section{Extension to fibred categories}

Motivated by the fact that the (categorical) semantics of second-order languages require fibred categories, we indicate in this section how our main Theorem 1 can be extended to this context. These results will be applied in Hoofman and Moerdijk (1994). We assume that the reader is familiar with the notions of fibred category and cartesian functor (see e.g. Bénabou (1985)).

Let $\mathrm{C}$ be a fixed category, and let $\mathrm{Fib}(\mathrm{C})$ be the 2-category of fibred categories over $\mathrm{C}$. So its objects are (Grothendieck) fibrations $P: \mathrm{E} \rightarrow \mathrm{C}$, its arrows are cartesian functors over $C$, and its 2-cells are vertical natural transformations (all as usual). Say a fibred category $P: \mathrm{E} \rightarrow \mathrm{C}$ has fibrewise splittings iff each category $\mathrm{E}_{B}=P^{-1}(B)$ has splittings. Denote by $\mathrm{Fib}_{v i}(\mathrm{C})$ the full 2-subcategory of $\mathrm{Fib}(\mathrm{C})$ whose objects have fibrewise splittings (where vi stands for vertical idempotent).

For a fibred category $P: \mathrm{E} \rightarrow \mathrm{C}$, we construct a new fibred category $\mathscr{K}_{v} \mathrm{E} \rightarrow \mathrm{C}$ as follows. The objects in $\mathscr{K}_{v} \mathrm{E}$ are pairs $\langle E, p\rangle$, where $E$ is an object in $\mathrm{E}$ and $p: E \rightarrow E$ is a vertical idempotent: $P(p)=i d_{P E}$ and $p^{2}=p$. The arrows $\langle E, p\rangle \rightarrow\left\langle E^{\prime}, p^{\prime}\right\rangle$ in $\mathscr{K}_{v} \mathrm{E}$ are arrows $f: E \rightarrow E^{\prime}$ in $\mathrm{E}$ satisfying $p^{\prime} \circ f \circ p=f$. The composition and identities for $\mathscr{K}_{v} \mathrm{E}$ are obvious (just as for the ordinary $\mathscr{K}$ ). Finally, we extend the functor $P: \mathrm{E} \rightarrow \mathrm{C}$ to a functor $\mathscr{K}_{v} \mathrm{E} \rightarrow \mathrm{C}$ by $\langle E, p\rangle \mapsto P E$ on objects and in the obvious way on arrows. We call $\mathscr{K}_{v}(P)$ the vertical Karoubi envelope of $P$.

Proposition 6. The functor $\mathscr{K}_{v} \mathrm{E} \rightarrow \mathrm{C}$ defines a fibred category with fibrewise splittings.

Proof. Let $u: B^{\prime} \rightarrow B$ be an arrow in $C$ and $\langle E, p\rangle$ be an object in $\mathscr{K}_{v}$ E with $P E=B$. We indicate how to define a cartesian lifting $u^{\#}:\left\langle E^{\prime}, p^{\prime}\right\rangle \rightarrow\langle E, p\rangle$ of $u$, and leave further details to the reader. First, choose a cartesian lifting $u^{b}: E^{\prime} \rightarrow E$ of $u$ in E. Then, since $\mathrm{E}$ is fibred over $\mathrm{C}$, there is a unique $p^{\prime}: E^{\prime} \rightarrow E^{\prime}$ with $u^{b} \circ p^{\prime}=u^{b}$. By uniqueness, $p^{\prime}$ is idempotent. Finally, take $u^{\#}=p \circ u^{b}:\left\langle E^{\prime}, p^{\prime}\right\rangle \rightarrow\langle E, p\rangle$ as the desired cartesian lifting of $u$ in $\mathscr{K}_{v} \mathrm{E}$.

It is easy to see that if the fibred category $E \rightarrow C$ is defined from an indexed category $\mathrm{C}^{o p} \rightarrow$ Cat, then $\mathscr{K}_{v} \mathrm{E} \rightarrow \mathrm{C}$ is induced from the composite indexed category $\mathrm{C}^{o p} \rightarrow \mathrm{Cat} \stackrel{\mathscr{K}}{\rightarrow}$ Cat.

Many properties of fibred categories are preserved by the vertical Karoubi envelope. For models of second order languages, the most relevant ones are:

(1) If $\mathrm{E} \rightarrow \mathrm{C}$ is fibrewise cartesian closed, then so is $\mathscr{K}_{v} \mathrm{E} \rightarrow \mathrm{C}$.

(2) If $\mathrm{E} \rightarrow \mathrm{C}$ is complete (cocomplete), then so is $\mathscr{K}_{v} \mathrm{E} \rightarrow \mathrm{C}$.

(For this last preservation property, recall from Bénabou (1985) that $P: E \rightarrow C$ is said to 
be complete (cocomplete) iff $\mathrm{C}$ has pullbacks and if, for each morphism $u$ in $\mathrm{C}$, the functor $u^{*}=P(u)$ has a right adjoint (left adjoint) satisfying the Beck-Chevalley condition.)

Let $P: \mathrm{E} \rightarrow \mathrm{C}$ and $P^{\prime}: \mathrm{E}^{\prime} \rightarrow \mathrm{C}$ be fibred categories over $\mathrm{C}$. A semi-functor $F: \mathrm{E} \rightarrow \mathrm{E}^{\prime}$ over $\mathrm{C}$ (that is, $P^{\prime} \circ F=P$ ) induces an ordinary functor $\mathscr{K}_{v}(F): \mathscr{K}_{v} \mathrm{E} \rightarrow \mathscr{K}_{v} \mathrm{E}^{\prime}$, exactly as in Section 2. Furthermore, a natural transformation $\alpha: F \rightarrow G$ between two such semi-functors induces an ordinary natural transformation $\mathscr{K}_{v}(\alpha): \mathscr{K}_{v}(F) \rightarrow \mathscr{K}_{v}(G)$. Say that $F$ is semi-cartesian iff $\mathscr{K}_{v}(F)$ is cartesian, and say that $\alpha$ is vertical iff $P\left(\alpha_{E}\right)=i d_{F E}$ for all $E \in \mathrm{E}$. This defines a 2-category $\mathrm{Fib}_{s}(\mathrm{C})$ with fibred categories as objects, cartesian semi-functors as 1-cells, and vertical natural transformations between such semi-functors as 2-cells. The following result is now a straightforward extension of Theorem 1.

Theorem 7. The vertical Karoubi envelope defines an equivalence of 2-categories

$$
\mathscr{K}_{v}: \mathrm{Fib}_{s}(\mathrm{C}) \rightarrow \mathrm{Fib}_{v i}(\mathrm{C}) .
$$

Remark. For a semi-functor $F: E \rightarrow E^{\prime}$ over $C$ as above, the property of being semicartesian can be described explicitly as follows. The semi-functor $F$ is semi-cartesian iff for every cartesian arrow $f: D \rightarrow E$ in $E$, its image $F(f)$ has the following property: $F(f)$ can be factorized uniquely (up to isomorphism) as $g \circ h$, where $h: F D \rightarrow K$ vertical and $g: K \rightarrow F E$ cartesian (for this factorization there exists a vertical $h^{\prime}: K \rightarrow F D$ so that $h^{\prime} \circ h=F\left(i d_{D}\right)$ and $\left.g \circ h \circ h^{\prime}=F\left(i d_{E}\right) \circ g\right)$.

Remark. Several of the results in this section can be further extended. For example, Proposition 6 above can be strengthened as follows. Let $u: B^{\prime} \rightarrow B$ be an arrow in C. A semi-cartesian arrow over $u$ is an arrow $f: E_{1} \rightarrow E$ over $u$ (that is, $P(f)=u$ ) equipped with an operation assigning to each arrow $g: E_{2} \rightarrow E$ and factorization $P(g)=u \circ v$ an arrow $\bar{g}: E_{2} \rightarrow E_{1}$ over $v$ satisfying $f \circ \bar{g}=g$. Moreover, we require the operation to be natural in the sense that $\overline{\mathrm{g}} \circ h=\overline{\mathrm{g} \circ h}$, for each $h: D \rightarrow E_{2}$. A functor $P: \mathrm{E} \rightarrow \mathrm{C}$ is a semi-fibred category iff for each $u: B^{\prime} \rightarrow B$ in $C$ and $E \in \mathrm{E}_{B}$, there exists a semi-cartesian morphism over $u$ with codomain $E$. Note that each fibred category is a semi-fibred category. We leave it to the reader to check the following proposition: the functor $\mathscr{K}_{v} \mathrm{E} \rightarrow \mathrm{C}$ defines a fibred category iff $E \rightarrow C$ is a semi-fibred category.

\section{References}

Bénabou, J. (1985) Fibred Categories and the Foundations of Naive Category Theory. J. Symb. Log. 50 10-37.

Freyd, P. A. and Scedrov, A. (1989) Categories, Allegories, North-Holland, Amsterdam.

Hayashi, S. (1985) Adjunction of Semifunctors: Categorical Structures in Non-Extensional LambdaCalculus. Theor. Comput. Sci. 41 95-104.

Hoofman, R. (1992) Non-Stable Models of Linear Logic. In: Nerode, A. and Taitslin, M. (eds.) Logical Foundations of Computer Science. Springer-Verlag Lecture Notes in Computer Science $620209-220$.

Hoofman, R. (1993a) The Theory of Semi-Functors. Mathematical Structures in Computer Science 3 93-128.

Hoofman, R. (1993b) Information Systems as Coalgebras, ILLC Prepublication Series, ML-92-08, University of Amsterdam. 
Hoofman, R. and Schellinx, H. (1991) Collapsing Graph Models by Preorders. In: Pitt, D. H., Curien, P.-L., Abramsky, S., Pitts, A. M., Poigné, A. and Rydeheard, D. E. (eds.) Category Theory and Computer Science. Springer-Verlag Lecture Notes in Computer Science 530 53-73.

Hoofman, R. and Moerdijk, I. (1994) On the Extensionalization of Second-Order Models (manuscript).

Jacobs, B. (1991) Semantics of the Second Order Lambda Calculus. Mathematical Structures in Computer Science $1327-360$.

Koymans, C. P. J. (1982) Models of the Lambda Calculus. Information and Control 52 306-322.

Kelly, G. M. and Street, R. (1974) Review of the Elements of 2-Categories, Category Seminar. Springer-Verlag Lecture Notes in Mathematics 420 75-103.

Lambek, J. and Scott, P. J. (1986) Introduction to Higher Order Categorical Logic, Studies in Advanced Mathematics 7, Cambridge University Press.

Mac Lane, S. (1971) Categories for the Working Mathematician, Springer-Verlag, New York.

Martini, S. (1987) An Interval Model for Second Order Lambda Calculus. In: Pitt, D. H., Poigné, A. and Rydeheard, D. (eds.) Category Theory and Computer Science. Springer-Verlag Lecture Notes in Computer Science $283219-237$.

Martini, S. (1992) Categorical Models for Non-Extensional $\lambda$-Calculi and Combinatory Logic. Mathematical Structures in Computer Science 2 327-357.

Román, L. (1989) On Partial Cartesian Closed Categories. In : Gray, J. W. and Scedrov, A. (eds.) Categories in Computer Science and Logic. Contemporary Mathematics 92, American Mathematical Society 349-356.

Seely, R. A. G. (1987) Categorical Semantics for Higher Order Polymorphic Lambda Calculus. J. Symb. Logic. 52 145-156.

Street, R. (1972) The Formal Theory of Monads. Journal of Pure and Applied Algebra 2 149-168. 